\title{
What factors influence community-dwelling older people's intent to undertake multifactorial fall prevention programs?
}

This article was published in the following Dove Press journal:

Clinical Interventions in Aging

26 November 2014

Number of times this article has been viewed

\section{Keith D Hill',2 \\ Lesley Day ${ }^{3}$ \\ Terry P Haines ${ }^{4,5}$}

'School of Physiotherapy and Exercise Science, Faculty of Health Sciences,

Curtin University, Perth, WA, Australia; ${ }^{2}$ National Ageing Research Institute, Royal Melbourne Hospital, Parkville, VIC, Australia; ${ }^{3}$ Falls Prevention Research Unit, Monash Injury Research Institute, Monash University, VIC, Australia; ${ }^{4}$ Allied Health Research Unit, Southern Health, Cheltenham, VIC, Australia; ${ }^{5}$ hyssiotherapy Department, Faculty of Medicine, Nursing, and Health Sciences, Monash University, VIC, Australia

Video abstract

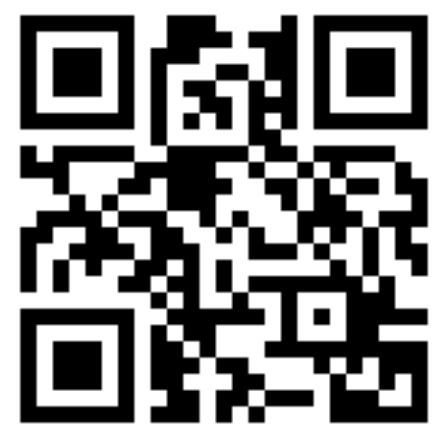

Point your SmartPhone at the code above. If you have a QR code reader the video abstract will appear. Or use:

http://dvpr.es/lud504N

Correspondence: Keith D Hill School of Physiotherapy and Exercise Science, Faculty of Health Sciences, Curtin University, GPO Box UI987, Perth, WA 6845, Australia

$\mathrm{Tel}+6 \mid 892663618$

Fax +6I 892663699

Email keith.hill@curtin.edu.au
Purpose: To investigate previous, current, or planned participation in, and perceptions toward, multifactorial fall prevention programs such as those delivered through a falls clinic in the community setting, and to identify factors influencing older people's intent to undertake these interventions.

Design and methods: Community-dwelling people aged $>70$ years completed a telephone survey. Participants were randomly selected from an electronic residential telephone listing, but purposeful sampling was used to include equal numbers with and without common chronic health conditions associated with fall-related hospitalization. The survey included scenarios for fall prevention interventions, including assessment/multifactorial interventions, such as those delivered through a falls clinic. Participants were asked about previous exposure to, or intent to participate in, the interventions. A path model analysis was used to identify factors associated with intent to participate in assessment/multifactorial interventions.

Results: Thirty of 376 participants (8.0\%) reported exposure to a multifactorial falls clinic-type intervention in the past 5 years, and $16.0 \%$ expressed intention to undertake this intervention. Of the 132 participants who reported one or more falls in the past 12 months, over one-third were undecided or disagreed that a falls clinic type of intervention would be of benefit to them. Four elements from the theoretical model positively influenced intention to participate in the intervention: personal perception of intervention effectiveness, self-perceived risk of falls, selfperceived risk of injury, and inability to walk up/down steps without a handrail $(P<0.05)$.

Conclusion: Multifactorial falls clinic-type interventions are not commonly accessed or considered as intended fall prevention approaches among community-dwelling older people, even among those with falls in the past 12 months. Factors identified as influencing intention to undertake these interventions may be useful in promoting or targeting these interventions.

Keywords: falls prevention, falls clinics, older adults, motivation

\section{Introduction}

Falls among older people remain a major public health problem and one that continues to contribute to growing use of health care services such as emergency department and hospital inpatient care, ${ }^{1}$ despite a strong evidence base for prevention developed over the past 15 years. ${ }^{2}$ The most recent Cochrane review of fall prevention in communityliving older people included 79,193 participants in 159 randomized trials, and identified that a range of single, multiple (limited suite of interventions for all participants), and multifactorial (multiple interventions are applied, based on individual need) interventions were effective in reducing falls and falls risk. ${ }^{2}$

There are a number of different ways to deliver multifactorial fall prevention interventions. However, a key feature is that these are "interventions in which 
two or more sub-domains of intervention can be given to participants, but the interventions are linked to each individual's risk profile"3 ( $p$ 19), often based on some form of risk assessment of the individual. One approach that has been utilized involves a health professional undertaking an assessment, often involving use of a falls risk assessment tool, and then tailoring the intervention to address identified risk factors. ${ }^{4,5}$ Another increasingly common approach is to utilize a multidisciplinary assessment of key falls risk factors through specialist falls clinics for older people. Falls clinics vary in their staffing, assessment procedures, level and type of interventions available, and duration of interventions. $^{6}$ A number of pre-post design studies have reported positive outcomes for falls clinics, including 50\% or more reductions in falls and fall injuries, and improved functional, balance, and mobility outcomes. ${ }^{7-10}$ Only one recent randomized trial of a falls clinic approach has been reported, showing a significant reduction in falls and fall-induced injuries in a specialist clinic in Finland. ${ }^{11}$ This program involved assessments by a physician, nurse, and physiotherapist and then a range of tailored programs instituted based on assessment findings, with a focus on medical review, strength and balance exercises, review of medication, review of home environments and safety modifications, and prescription of vitamin $\mathrm{D}$ and calcium when indicated.

While multifactorial interventions such as multidisciplinary fall clinics that incorporate individual fall risk assessment and targeted interventions have a growing evidence base supporting their effectiveness in reducing falls and falls risk, an important factor constraining the degree of effectiveness of these approaches is limited uptake and adherence to recommended interventions by older people receiving these services. For example, in an Australian study of older people presenting to 14 falls clinics, adherence varied between the different individual recommendations, with $74 \%$ of older people fully adhering to further medical investigations and medication change, 53\% fully adhering to exercise interventions, and only $16 \%$ fully adhering to recommendations to use hip protectors. ${ }^{7}$

In order to optimize outcomes associated with multifactorial intervention programs aiming to reduce falls among older people - such as falls clinics - there is a need to more fully understand factors influencing participation in this type of intervention. According to the Theory of Planned Behavior, intention is a key antecedent of behavior change and is therefore likely to be an important factor predicting participation in falls interventions. ${ }^{12}$ The aims of this study were 1) to investigate older people's previous, current, or planned participation in, and perceptions toward, falls and balance clinic interventions, and 2) to identify factors influencing older people's intent to undertake multifactorial fall prevention programs such as those delivered through a falls and balance clinic approach in the community setting.

\section{Materials and methods}

This study was undertaken as one part of a multicomponent project aiming to implement evidence-based, targeted falls prevention programs for older people in Victoria, Australia. ${ }^{13}$ The four components included 1) data analysis of older people admitted to hospital due to falls, and factors associated with long length of stay; ${ }^{14}$ 2) a phone survey of older people about their participation, and intent to participate in future, in evidence-based fall prevention interventions; ${ }^{15,16} 3$ ) identification of government departments and programs with potential to value add to current and future planned fall prevention programs; ${ }^{16,17}$ and 4) development and pilot evaluation of sustainability guidelines for fall prevention programs (http://www. monash.edu.au/miri/research/research-areas/home-sportand-leisure-safety/fpru/nhmrc guidelines and workbook. pdf). This study reports a subset of the results of the second component - the telephone survey.

We applied the Health Belief Model ${ }^{18}$ with additional constructs from the Protection Motivation Theory ${ }^{19}$ and the Theory of Planned Behavior ${ }^{12}$ to construct a model to explain the process by which individuals form an intention to undertake falls prevention interventions. ${ }^{13}$ Constructs added to the Health Belief Model include extra components contributing to the benefits associated with undertaking the intervention (including self-efficacy to undertake intervention, non-fallrelated benefits, and importance to the individual of preventing harm from falling) and components contributing to the costs associated with undertaking the intervention (direct and indirect costs, and conflicts with social norms). ${ }^{13}$ The model predicts factors that would be associated with intention to undertake falls prevention interventions.

Inclusion criteria for participation in the study were being aged $\geq 70$ years; living in the state of Victoria, Australia; community dwelling; being cognitively intact (score $\leq 12$ on the six-item cognitive impairment test, score range 0-28; higher score indicates greater cognitive impairment); ${ }^{20}$ and having sufficient spoken English language proficiency to participate (as judged by the telephone interviewer). The sampling frame for the study was the 2006 electronic residential telephone listings (the most readily available and affordable at the time). Participants were recruited by a survey research company that phoned randomly selected households, 
screened respondents for all eligibility criteria except cognitive status, ascertained their medical conditions (respondents were read a list of 16 medical conditions associated with increased risk of falls and asked "Have you ever been told by a doctor or a nurse that you have xxxx condition?"), and ascertained their willingness to participate in a survey about health services that have been shown to reduce the risk of falls. Sampling was modified so that equal numbers of people with and without one of the following chronic medical conditions were recruited: diabetes, congestive heart failure, pulmonary disease, renal disease, depression, or anxiety, as these conditions have been shown to be associated with increased hospital length of stay for older people admitted due to a fall. ${ }^{14}$ The research team subsequently contacted the eligible participants, screened for cognitive impairment, and administered the questionnaire. Data were collected by telephone interview by trained research assistants between December 2010 and February 2011.

Participant flow through the study is reported in Figure 1. In total, 554 people consented to be contacted by the telephone research team, and 394 (71\%) commenced the survey. Main reasons for nonparticipation included refusal after study requirements were described, unable to make contact after initial consent, and presence of cognitive impairment.

The survey was modified from questionnaires utilized previously to investigate perceived risk of falls and perceptions about participation in falls prevention interventions of older people discharged from hospital. ${ }^{21-23}$ Following an initial section on demographic and fall history questions (World Health Organization definition of fall used "A fall is an event which results in a person coming to rest inadvertently on the ground or floor or other lower level." ${ }^{24}$ [p 1]) and questions about their own perception of their falls risk, the survey had five subsequent components. Each involved a detailed description (in lay terms) of five evidence-based falls prevention interventions (group exercise, home-based exercise, home assessment and modifications, psychotropic medication review, and multifactorial interventions). These interventions are recommended by the Cochrane Collaboration review of interventions to prevent falls in the community

13,614 telephone numbers selected

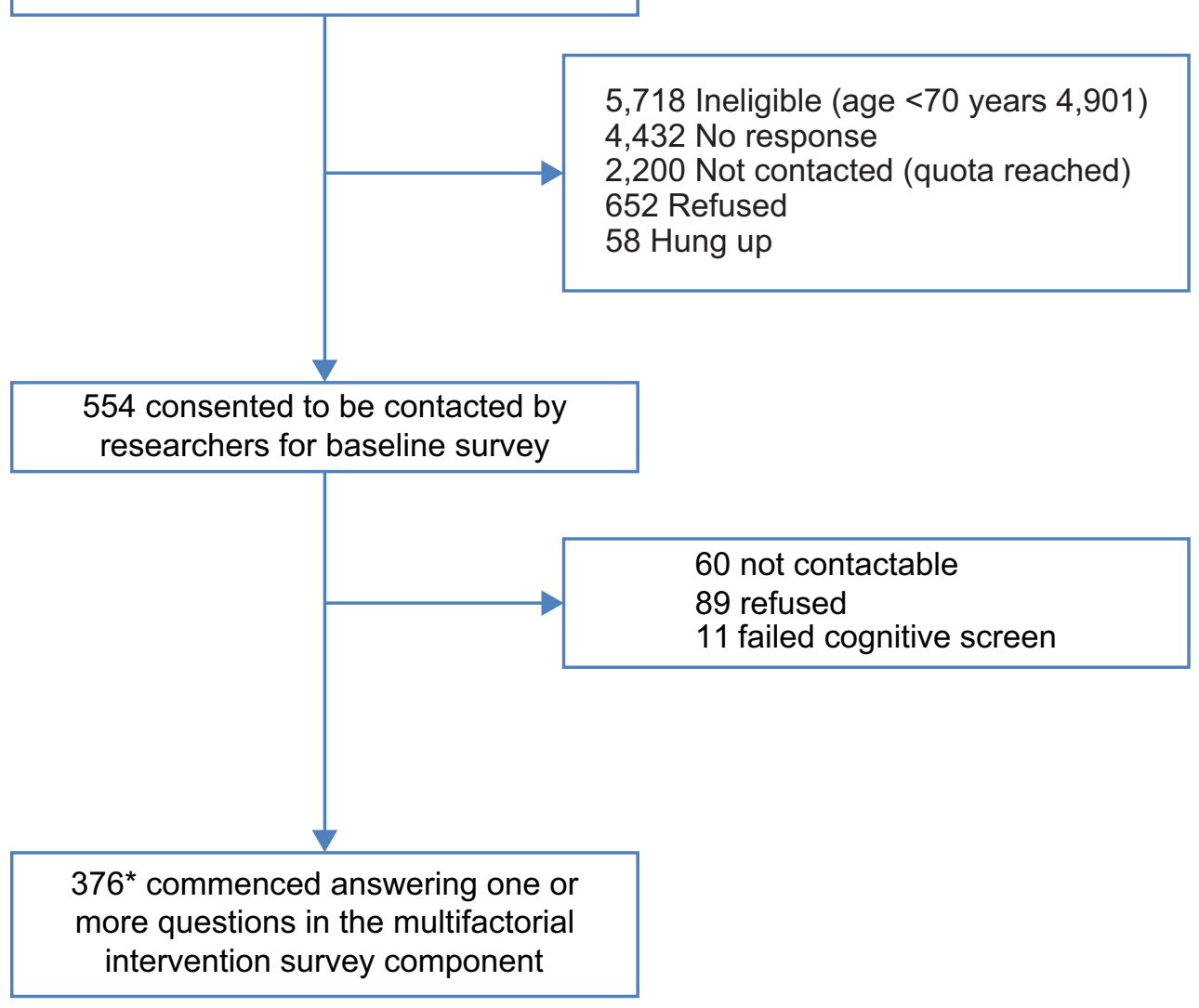

Figure I Participant flow through the study.

Note: $* 394$ participants commenced participation in the overall survey, but $4.6 \%(n=18)$ ceased participation prior to reaching the component relating to the multifactorial intervention, leaving 376 commencing the multifactorial component of the survey. 
setting $^{2}$ and were also the ones determined by the research team to be most applicable to the community setting. ${ }^{17}$ For the five intervention types, a detailed written description and appropriate visual images were posted to participants to review before the telephone survey. During the telephone survey, the interviewer went through the detailed description of each intervention prior to questions about that intervention, to ensure the respondent understood the type of intervention being described, including details about the likely location, travel time, time of day, and out-of-pocket expenses associated with the intervention (see Table 1 for details provided for the multifactorial intervention). The order of presentation of the five interventions was randomized for each participant.

Participants were asked about their previous, current, or planned participation in, and perceptions toward, the five selected falls prevention interventions through a range of questions using a five-point Likert scale (strongly agree to strongly disagree with undecided as a central point). The participation questions asked respondents if they had previously participated in or received the intervention in the past 5 years, or if they were still participating in the intervention. Open-ended questions were also used to explore some aspects of questions relating to the five intervention types. In total, the initial survey took, on average, approximately 60 minutes.

For the section of the survey investigating "general effectiveness of intervention", the item wording was "I think if a

Table I Description provided (by post and by the interviewer) to detail what an assessment and multifactorial intervention (such as a falls clinic) would be like

In this kind of intervention, you would be given an appointment at a falls and balance clinic at a hospital that would likely take you no more than

45 minutes to travel to by your usual means of transportation if you live in a metropolitan area and no more than 2 hours if you live in a regional area. A geriatrician, occupational therapist, and physiotherapist who specialize in falls and balance problems would conduct an assessment with you, and you would then have a 6-week and 6-month reassessment. They would make recommendations on how you can decrease your risk of falling and improve your balance. Examples of some recommendations may be:

- for you to attend a weekly exercise program;

- to have a home visit by an occupational therapist to advise on home modifications;

- to recommend changes to your medications or footwear. These suggestions would be explained to you and written down for you. The initial appointment would take between 2 and 3 hours, and the follow-up appointments approximately I hour. Attending each assessment would cost AUD $\$ 10$ out of your own pocket, which would be $\$ 30$ to attend all three sessions. There may be further costs associated with some recommendations that may be made (eg, attending a group exercise program) that would be additional to this. man/woman (of same sex as respondent) about my age were to participate in a ... (the falls prevention intervention) like this, it would decrease their risk of falling" (see Figure 2). For the domain of "personal effectiveness of intervention if undertaken", the item wording used was "I think if I were to participate in a ... (the falls prevention intervention) like this, it would decrease my risk of falling." For the domain of "intention to undertake intervention", the item wording used was "I will start participating in a ... (the falls prevention intervention) like this within the next 6 months." These items were rephrased slightly depending on whether the participant was currently participating in or had previously received the falls prevention intervention. For example, those currently participating in a multifactorial fall prevention program had the item wording for the latter domain altered to "I will continue participating in a multifactorial fall prevention program like this for the next 6 months."

The focus of this paper is to report results of the survey associated with the multifactorial (falls clinic-type) intervention. Analyses for intention to undertake the other interventions investigated in the survey are being reported separately.

The study was approved by the Monash University Human Ethics Research Committee.

\section{Statistical analysis}

Demographic data were reported for the full sample and the subgroups participating in or expressing intent in undertaking the multifactorial assessment and intervention program, using mean (standard deviation) for normally distributed continuous data, or median (interquartile range) for ordinal or nonnormally distributed continuous data.

A path model development approach was used to identify factors with direct ( \pm indirect) effects on the intention to participate in a falls prevention assessment and multifactorial intervention program over the following year. Path models allow a deeper understanding of data structure than traditional multiple regression approaches, as they permit identification of both direct effects between indicator and response variables and indirect effects that act through mediating variables. In our model building, we retained indicator variables if they had a significant $(\alpha=0.05)$ direct effect on the response variable, while significant indirect effects were also retained in the model if they were mediated through another response variable that itself had a significant direct effect on the response variable. A Bayesian Markov chain Monte Carlo (McMC) approach (AMOS version 19.0) was used, as our response variables had ordinal and binary response scaling. 


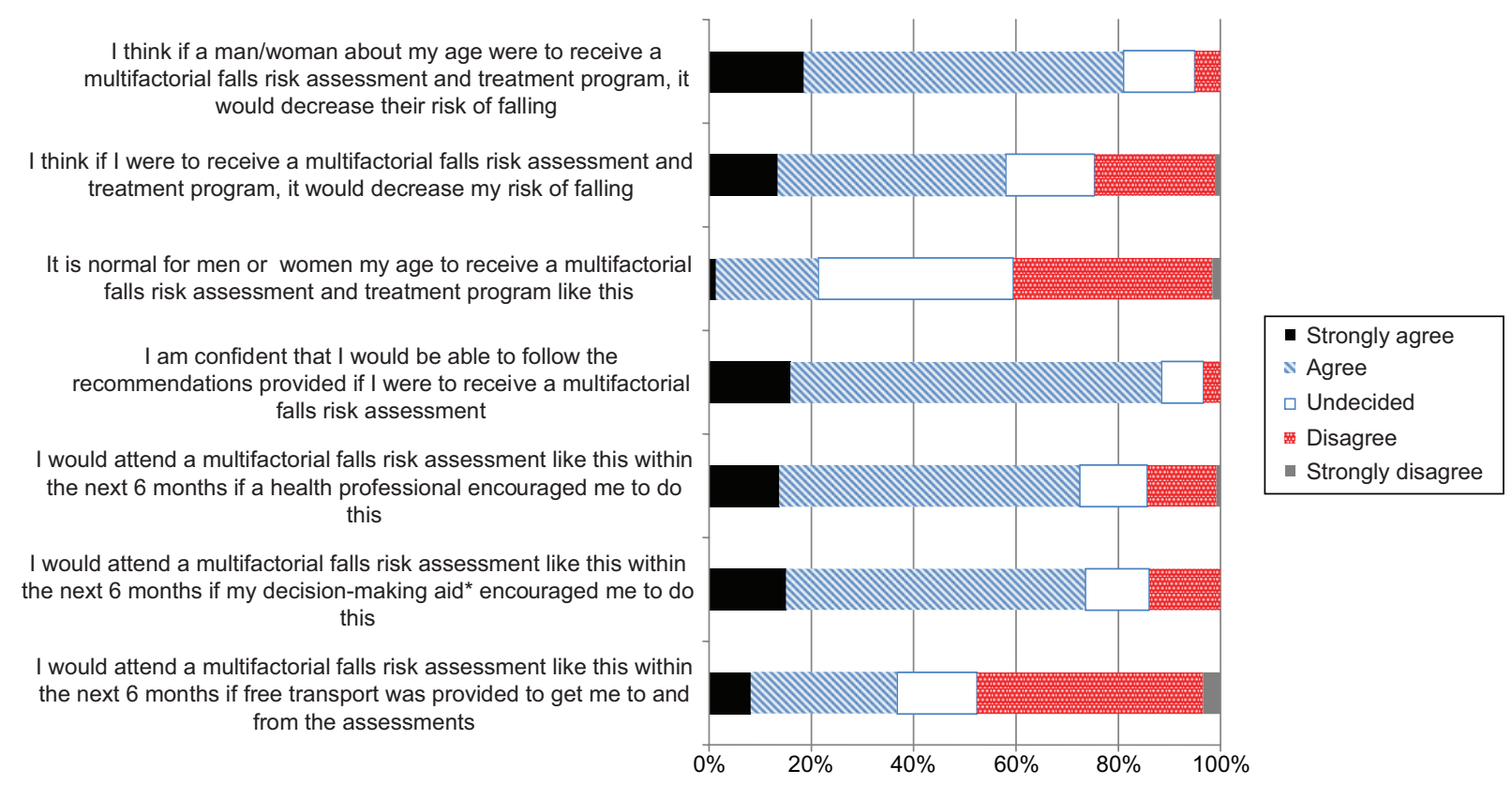

Figure 2 Responses to questions on initial survey regarding perceptions and intent to participate in a multifactorial fall prevention program.

Note: *Decision-making aid is the person/s who are most involved in how an individual makes decisions about their health (may be self or others).

Our model building commenced with inclusion of variables measuring domains from our theoretical mode ${ }^{13}$ with direct effects on the response variable. For the model, intention to undertake a falls assessment and multifactorial intervention at a falls and balance clinic was the response variable. Thus, selfperceived risk of falling, self-perceived risk of harm, perception of effectiveness of this intervention in general, perception of personal effectiveness of this intervention, confidence in ability to follow the recommendations of a falls assessment, and whether the participant considered this intervention to be a social norm were the indicator variables that commenced in the model. Motivation to prevent falls was not included, as $>95 \%$ of respondents either agreed or strongly agreed with this item. Pairwise polychoric correlations (calculated using STATA I/C version 11.0) were examined to determine whether indirect effects likely to be mediated through other factors within the model could be identified. Once the starting theoretical model had been reduced, other demographic variables were introduced to the model, and theoretical indicator variables were reintroduced to determine whether readdition of these variables following addition of demographic indicator variables, changed whether these variables should be retained. The process of removing nonsignificant factors and then re-examining remaining factors was continued until all factors within the path model had significant direct effects with the outcome and there were no further factors to consider for inclusion.

When examining the model, random walks using the McMC approach continued until the model converged, using a threshold of 1.001. Standardized effects of indicator on response variables (equivalent to standardized regression coefficients) were calculated along with their $95 \%$ confidence intervals. Model fit was examined using the posterior predictive $P$ (values closer to 0.50 reflect better fit). Where model fit was questionable, graphical diagnostic plots were examined to identify if McMC samples converged to the posterior distribution. Both polygon plots examining first and third samples and trace plots were examined.

\section{Results}

The demographics of study participants who completed the survey are presented in Table 2. A total of 394 participants commenced the overall survey. However, 18 (4.6\%) did not reach the stage of commencing the multifactorial intervention component, leaving 376 participants with data on this intervention. A total of $30(8.0 \%)$ of these 376 participants indicated that they had been exposed to this type of intervention in the past 5 years (Table 2). Thirty-five percent of the full sample reported having had one or more falls in the 12 months prior to the survey, while 19 of the 30 participants who reported that they had undergone a multifactorial fall prevention program in the past 5 years had one or more falls in this preceding 12-month period.

Sixty respondents $(16.0 \%)$ reported that they intended to undertake a multifactorial fall prevention program in the next 6 months ( $\mathrm{n}=11$ strongly agreed, $\mathrm{n}=49$ agreed). 
Table 2 Comparison of characteristics of I) participants completing the multifactorial component of the survey, 2) the subgroup of these participants who have previously participated in a multifactorial fall prevention intervention, and 3) the subgroup with intent to participate in a multifactorial fall prevention intervention

\begin{tabular}{|c|c|c|c|}
\hline & $\begin{array}{l}\text { All respondents } \\
\text { who completed } \\
\text { multifactorial } \\
\text { component } \\
\text { of survey }(n=376)\end{array}$ & $\begin{array}{l}\text { Respondents who had } \\
\text { done a multifactorial } \\
\text { fall prevention } \\
\text { intervention in past } \\
5 \text { years }(n=30)\end{array}$ & $\begin{array}{l}\text { Respondents with } \\
\text { intention to do a } \\
\text { multifactorial fall } \\
\text { prevention } \\
\text { intervention }(n=60)\end{array}$ \\
\hline Age, years - mean (SD) & $77.7(5.8)$ & $78.9(5.2)$ & $76.9(5.8)$ \\
\hline Female sex $-\mathrm{n}(\%)$ & $223(58.8)$ & $20(66.6)$ & $4 I(68.3)$ \\
\hline Living alone $-\mathrm{n}(\%)$ & $190(50.5)$ & $18(60.0)$ & $31(51.7)$ \\
\hline \multicolumn{4}{|l|}{ Chronic health conditions } \\
\hline Arthritis & $215(57.2)$ & $19(63.3)$ & $38(63.3)$ \\
\hline Heart disease (other than CHF) & $106(28.0)$ & $5(16.7)$ & $17(28.3)$ \\
\hline Cancer & $88(23.4)$ & $5(16.7)$ & $14(23.3)$ \\
\hline Visual impairment (other than cataract) & $87(23.1)$ & $6(20.0)$ & $14(23.3)$ \\
\hline Osteoporosis & $84(22.3)$ & II (36.7) & $17(28.3)$ \\
\hline Cataracts & $80(21.3)$ & $5(16.7)$ & $14(23.3)$ \\
\hline Diabetes & $61(16.1)$ & $5(16.7)$ & $6(10.0)$ \\
\hline Depression/anxiety & $60(16.0)$ & $5(16.7)$ & $15(25.0)$ \\
\hline Number of prescription medications - median (IQR) & $3(1.5,4.5)$ & $4.5(2.0,7.0)$ & $3.5(1.5,5.5)$ \\
\hline $\begin{array}{l}\text { Emergency department presentation for fall in past } 6 \text { months } \\
\text { (not admitted) }-\mathrm{n}(\%) \text { ( } 372 \text { responses) }\end{array}$ & $10(2.6)$ & $4(13.3)$ & $3(5.0)$ \\
\hline Admitted to hospital in past 6 months $>$ I night $-\mathrm{n}(\%)$ (372 responses) & $73(19.6)$ & $9(30.0)$ & $14(23.3)$ \\
\hline Retrospective recall of one or more falls in preceding 12 months $-\mathrm{n}(\%)$ & $132(35.1)$ & $19(63.3)$ & $24(40.0)$ \\
\hline
\end{tabular}

Abbreviations: SD, standard deviation; CHF, chronic heart failure; IQR, interquartile range.

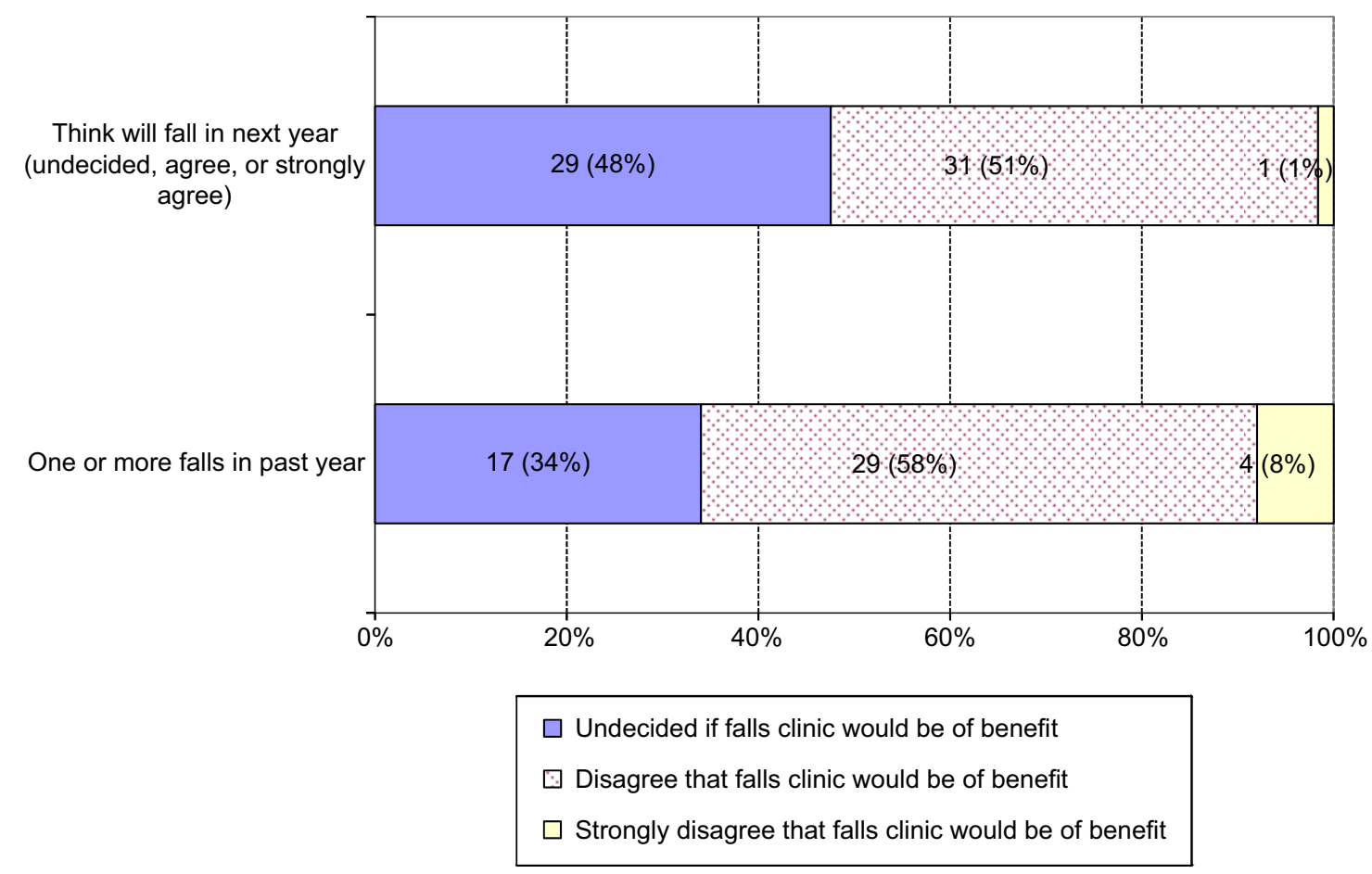

Figure 3 Number of participants who reported they did not consider the falls clinic type of intervention would be of personal benefit, among those who I) fell in the past 12 months and 2) thought they were likely to fall in the next 12 months.

Notes: Percentages reported are for the proportion of participants at increased risk of falls due to I) reporting that they thought they would fall in the next 12 months and 2) having had one or more falls in the past 12 months ( $n=132$ ). The figure does not include I) the participants who did not think they would fall in the next 12 months and 2 ) the participants who did not fall in the past 12 months. 
Figure 2 reports responses on the five-point Likert scale relating to perceptions about the value and possible participation in multifactorial interventions. The majority of survey respondents indicated they considered that a multifactorial intervention would be effective in reducing falls for men or women of their own age (strongly agree $18.6 \%$, agree $62.5 \%$ ), and that if they were to undertake a multifactorial falls assessment intervention, it would reduce their risk of falling (strongly agree $13.5 \%$, agree $44.5 \%$ ). However, there were a moderate number of participants who were undecided or who considered this intervention would not reduce their risk of falling, despite having fallen in the past 12 months, or reporting likelihood of falling in the next 12 months (Figure 3). Importantly, $72.5 \%$ indicated they would attend this type of intervention if encouraged by a health professional to do so (13.8\% strongly agree, $58.7 \%$ agree) and $73.6 \%$ if encouraged by their main decision-making aid (the person/s who are most involved in how an individual makes decisions about their health) (15.1\% strongly agree, $58.5 \%$ agree). Free transport was only considered a factor in attending this type of intervention for $36.8 \%$ (8.2\% strongly agree, $28.6 \%$ agree).

The results of the path analysis examining intention to participate in the falls assessment and multifactorial intervention are shown in Table 3. These data are unstandardized regression weights ( $95 \%$ confidence intervals) along with model diagnostic data, with larger weights indicating greater effect. The intention model posterior predictive $P$-value was not optimal. Therefore, diagnostic plots were examined. These plots demonstrated reasonable convergence to the posterior distribution for each effect examined in the model. Thus, the model was retained. This analysis demonstrates that three elements contained within our theoretical model significantly influenced intention to

Table 3 Unstandardized regression weight ( $95 \%$ confidence interval) of variables retained within the model for factors associated with intent to participate in a multifactorial fall prevention (falls clinic) program $(n=329)$

\begin{tabular}{ll}
\hline Indicator variable & $\begin{array}{l}\text { Response variable } \\
\text { (intention) }\end{array}$ \\
\hline $\begin{array}{l}\text { Perception of personal effectiveness of falls } \\
\text { assessment and multifactorial intervention } \\
\text { for the prevention of falls }\end{array}$ & $0.38(0.29,0.46)$ \\
Self-perceived risk of injury if one were to fall & $0.13(0.03,0.23)$ \\
Self-perceived risk of falls & $0.1 \mathrm{I}(0.03,0.19)$ \\
Inability to walk up and down steps without & $0.10(0.00,0.20)$ \\
a handrail & \\
Intention model & \\
Convergence statistic & $1.00 \mathrm{I}$ \\
Markov chain Monte Carlo random walks & $\mathrm{I} 7,330$ \\
Posterior predictive $P$ & 0.00 \\
\hline
\end{tabular}

undertake the falls assessment and multifactorial intervention. Perception of personal effectiveness of falls assessment and multifactorial intervention had the strongest association with intent. Self-perceived risk of falls had a positive direct effect on intention to participate, as did self-perceived risk of injury if one were to fall. In addition, inability to walk up and down steps without a handrail was borderline significant in its association with intention to participate in this intervention. None of the demographic or comorbidity data added a significant amount of additional information to the model.

\section{Discussion}

This study explored older people's exposure to, and perceptions of, multifactorial assessment and intervention programs such as a falls clinic. Although these services have become more common, ${ }^{6,7}$ and the growing evidence base supports their efficacy, our study reinforces that exposure of older people to these types of interventions is low ( $8 \%$ of our sample, although $35.1 \%$ of the sample reported one or more falls in the past 12 months). It should be noted that these types of interventions are relatively resource intense, often involving assessments by a number of medical and allied health professionals and several follow-up assessments or reviews, ${ }^{6}$ and so have been reported as being most appropriate for those at higher risk of falls or those for whom other single intervention approaches such as general practitioner review or home safety assessment in isolation have been insufficient to reduce an individual's risk of falling. Those who had reported exposure to a falls clinic in the past 5 years did report a substantially higher risk of falling in the past 12 months, suggesting it is those at high risk who have undertaken this type of program.

Several factors were identified as being associated with intent to undertake a falls clinic type of intervention in the next 12 months. The strongest of these was the perception of personal effectiveness of this type of intervention to reduce falls. This is consistent with a systematic review of facilitators and barriers to participation in falls prevention programs more generally, which concluded that information that falls are "preventable rather than unpredictable" and "countering the belief that nothing can be done for falls" was a valuable facilitator to undertaking falls prevention programs. ${ }^{25}$ In our study, while $58 \%$ of respondents considered this type of intervention would decrease their risk of falling, there were still a moderate number of people who had fallen in the past 12 months $(\mathrm{n}=50)$ or who considered they were likely to fall in the next 12 months $(\mathrm{n}=61)$ who were undecided or did not consider this type of intervention 
would reduce their risk of falling (Figure 3). Older adults have been found to infrequently discuss falls prevention with health professionals, and over a third of those who did discuss falls with a general practitioner did not remember the details of the discussion. ${ }^{15,26}$ Improved communication and education strategies by health practitioners in imparting relevant fall prevention messages to at-risk older people are needed, which may assist them in deciding to undertake this type of intervention. An alternative approach may be to use multimedia education programs that have been shown to improve falls threat knowledge, and to achieve increased fall prevention behavior change in community-dwelling older people. ${ }^{27}$ Inability to walk up and down stairs without a rail was a borderline significant factor associated with intent to undertake the falls clinic intervention. Inability to walk up and down stairs without a rail is likely to be a clear indicator to an older person of their reduced function that influences their decision making. There may be value for practitioners to emphasize the importance of this as a falls risk factor and the potential for some falls clinic interventions (eg, exercise) to also improve function such as walking up and down stairs, as well as potentially reducing risk of future falls.

Porter et a ${ }^{28}$ identified that among a sample of homebound older women who experienced falls, although intent to minimize risk of future falls was articulated, many of the women were uncertain about how to do this. Preventive strategies reported tended to be limited to trying to minimize the same falls risk circumstance occurring in future, rather than more broad perspectives on minimizing falls risk, including undergoing a falls risk assessment. Future research needs to improve our understanding of factors that will support older people, especially those at increased risk of falls, to consider undertaking and following through with recommended interventions from a falls clinic assessment.

Consistent with other studies, ${ }^{25,29}$ results from this study highlight that health professionals and family or others who assist in making health-related decisions appear to be in a strong position to influence at-risk older people to undertake a falls clinic type of intervention. However, even commencing this type of intervention is often not associated with high uptake of the various recommended interventions that can be made from an individualized assessment of falls risk, ${ }^{7}$ and so efforts need to be made by staff involved in these assessments and other key health professionals involved in care of these at-risk older people to support sustained engagement in these recommended interventions to maximize likely outcomes. Strategies such as a person-centered approach in which the older person is actively engaged in decision making about recommendations, ${ }^{25}$ goal setting, engaging with family, ${ }^{30}$ and prioritizing a limited number of interventions for those with many risk factors to be addressed ${ }^{30,31}$ may improve adherence to recommended interventions through falls clinic interventions.

There are several limitations of this study. Sampling response bias may limit the generalizability of results, although potential participants were initially randomly selected from the electronic telephone directory, with some additional purposive sampling. Although there may be some limitations of telephone surveys relative to face-to-face interviews, telephone surveys are more cost-effective for large samples and have been shown to provide similar results compared with face-to-face questioning in a study investigating falls risk and circumstances ${ }^{32}$ Study results may not be generalizable to other countries with different models of care and costs associated with falls clinic-type interventions. In addition, key measures in this study, such as intention to participate in a multifactorial intervention, and other data, such as falls history, were all based on self-report. Future research should extend this study to investigate factors associated with actual uptake and sustained engagement in this type of fall prevention activity. A strength of the study includes its theoretical underpinning.

\section{Conclusion}

Limited uptake of evidence-based fall prevention programs in the community can negatively influence the successful implementation of these interventions. This study has identified four factors associated with intent to undertake a multifactorial fall clinic type of intervention: personal perception of intervention effectiveness, self-perceived risk of falls, selfperceived risk of injury, and inability to walk up/down steps without a handrail. Focusing on these factors may be useful for health professionals in supporting at-risk older people considering engagement in this type of intervention.

\section{Acknowledgments}

We thank Professor Leon Flicker for his input into the selection of the cognitive screening measure, Dr Trang Vu for assistance with the background literature review, Noelene Deveson for coordinating the baseline survey, and our research assistants who collected the data. Role of the funding source: This study was undertaken as part of a Partnership grant (ID 546282) funded by the National Health and Medical Research Council (NHMRC) and the Department of Health, Victoria, Australia. The study sponsors did not have any involvement in the study design, data collection, analysis, or interpretation of data or in the writing of this manuscript and the decision to submit the manuscript for publication, for the reported study. Staff of the Department 
of Health, Victoria were members of the project team and contributed involvement to components of the Partnership study as investigators.

\section{Disclosure}

The authors report no conflicts of interest in this work.

\section{References}

1. Bradley C. Trends in hospitalisations due to falls by older people, Australia, 1999-2000 to 2010-2011. Injury Research and Statistics Series 84. Canberra: AIHW; 2013.

2. Gillespie L, Robertson M, Gillespie W, et al. Interventions for preventing falls in older people living in the community. Cochrane Database Syst Rev. 2012;9:CD007146.

3. Workpackage 1 of the Prevention of Falls Network Europe (ProFANE) Project. Manual for the Fall Prevention Classification System; 2007. Available from: http://www.profane.eu.org/documents/ Falls_Taxonomy.pdf. Accessed October 2, 2014.

4. Russell MA, Hill KD, Day LM, et al. A randomized controlled trial of a multifactorial falls prevention intervention for older fallers presenting to emergency departments. J Am Geriatr Soc. 2010;58(12):2265-2274.

5. Hogan DB, MacDonald FA, Betts J, et al. A randomized controlled trial of a community-based consultation service to prevent falls. CMAJ. 2001;165(5):537-543.

6. Hill K, Smith R, Schwarz J. Falls clinics in Australia: a survey of current practice, and recommendations for future development. Aust Health Rev. 2001;24(4):163-174.

7. Hill KD, Moore KJ, Dorevitch MI, Day LM. Effectiveness of falls clinics: an evaluation of outcomes and client adherence to recommended interventions. J Am Geriatr Soc. 2008;56(4):600-608.

8. Perell KL, Manzano ML, Weaver R, et al. Outcomes of a consult fall prevention screening clinic. Am J Phys Med Rehabil. 2006;85(11): 882-888.

9. Sze PC, Cheung WH, Lam PS, Lo HS, Leung KS, Chan T. The efficacy of a multidisciplinary falls prevention clinic with an extended step-down community program. Arch Phys Med Rehabil. 2008;89(7):1329-1334.

10. Alvord LS, Benninger MS, Stach BA. A preliminary study of the effectiveness of an otolaryngology-based multidisciplinary falls prevention clinic. Ear Nose Throat J. 2008;87(9):510-513.

11. Palvanen M, Kannus P, Piirtola M, Niemi S, Parkkari J, Jarvinen M. Effectiveness of the Chaos Falls Clinic in preventing falls and injuries of home-dwelling older adults: a randomised controlled trial. Injury. 2014;45(1):265-271.

12. Madden T, Ellen PS, Ajzen I. A comparison of the theory of planned behavior and the theory of reasoned action. Pers Soc Psychol Bull. 1992;18(1):3-9.

13. Day L, Finch CF, Hill KD, et al. A protocol for evidence-based targeting and evaluation of statewide strategies for preventing falls among community-dwelling older people in Victoria, Australia. Inj Prev. 2011;17(2):e3.

14. Vu T, Finch CF, Day L. Patterns of comorbidity in community-dwelling older people hospitalised for fall-related injury: a cluster analysis. $B M C$ Geriatr. 2011;11:45.
15. Lee D, Day L, Hill K, Clemson L, McDermott F, Haines T. What factors influence older adults to discuss falls with their healthcare providers? Health Expect. Epub 2013 Oct 13.

16. Day L, Trotter M, Hill K, Haines T, Thompson C. Implementation of evidence-based falls prevention in clinical services for high-risk clients. J Eval Clin Pract. 2014;20(3):255-259.

17. Day L, Donaldson A, Thompson C, Thomas M. Integration of proven falls prevention interventions into government policies and programs. Aust N Z J Public Health. 2014;38(2):122-127.

18. Rosenstock IM, Strecher VJ, Becker MH. Social learning theory and the Health Belief Model. Health Educ Q. 1988;15(2):175-183.

19. Taylor AH, May S. Threat and coping appraisal as determinants of compliance with sports injury rehabilitation: an application of Protection Motivation Theory. J Sports Sci. 1996;14(6):471-482.

20. Brooke P, Bullock R. Validation of a 6 item cognitive impairment test with a view to primary care usage. Int J Geriatr Psychiatry. 1999; 14(11):936-940.

21. Hill AM, Hoffmann T, Beer C, et al. Falls after discharge from hospital: is there a gap between older peoples' knowledge about falls prevention strategies and the research evidence? Gerontologist. 2011;51(5):653-662.

22. Hill AM, Hoffmann T, McPhail S, et al. Factors associated with older patients' engagement in exercise after hospital discharge. Arch Phys Med Rehabil. 2011;92(9):1395-1403.

23. Haines T, McPhail S. Threat appraisal for harm from falls: insights for development of education-based intervention. Open Longev Sci. 2011;5:1.

24. World Health Organization. WHO Global Report on Falls Prevention in Older Age. Geneva: WHO; 2007.

25. McInnes E, Askie L. Evidence review on older people's views and experiences of falls prevention strategies. Worldviews Evid Based Nurs. 2004;1(1):20-37.

26. Lee D, McDermott F, Hoffmann T, O’Connell B, Haines TP. "They will tell me if there is a problem": limited discussion between health professionals, older adults and their caregivers on falls prevention during and after hospitalization. Health Educ Res. Epub 2013 Sep 17.

27. Schepens SL, Panzer V, Goldberg A. Randomized controlled trial comparing tailoring methods of multimedia-based fall prevention education for community-dwelling older adults. Am J Occup Ther. 2011;65(6):702-709.

28. Porter EJ, Matsuda S, Lindbloom EJ. Intentions of older homebound women to reduce the risk of falling again. J Nurs Scholarsh. 2010;42(1):101-109.

29. Yardley L, Beyer N, Hauer K, McKee K, Ballinger C, Todd C. Recommendations for promoting the engagement of older people in activities to prevent falls. Qual Saf Health Care. 2007;16(3):230-234.

30. Hill K. Don't lose sight of the importance of the individual in effective falls prevention interventions. BMC Geriatr. 2009;9:13.

31. Campbell AJ, Robertson MC. Rethinking individual and community fall prevention strategies: a meta-regression comparing single and multifactorial interventions. Age Ageing. 2007;36(6):656-662.

32. Mackintosh S, Fryer C, Hill K. Telephone and face-to-face interviews generate similar falls circumstances information from community-dwelling adults with stroke. Aust $N$ Z J Public Health. 2009;33(3):295-296.
Clinical Interventions in Aging

\section{Publish your work in this journal}

Clinical Interventions in Aging is an international, peer-reviewed journal focusing on evidence-based reports on the value or lack thereof of treatments intended to prevent or delay the onset of maladaptive correlates of aging in human beings. This journal is indexed on PubMed Central, MedLine,

\section{Dovepress}

CAS, Scopus and the Elsevier Bibliographic databases. The manuscript management system is completely online and includes a very quick and fair peer-review system, which is all easy to use. Visit http://www.dovepress. com/testimonials.php to read real quotes from published authors. 